\title{
System Architecture for Autonomous Mobile Manipulation of Everyday Objects in Domestic Environments
}

\author{
Tarık Keleştemur \\ College of Engineering \\ Northeastern University \\ kelestemur@ece.neu.edu
}

\author{
Naoki Yokoyama \\ College of Engineering \\ Northeastern University \\ cho.na@husky.neu.edu
}

\author{
Joanne Truong \\ College of Engineering \\ Northeastern University \\ truong.j@husky.neu.edu
}

\author{
Anas Abou Allaban \\ College of Engineering \\ Northeastern University \\ abouallaban.a@husky.neu.edu
}

\author{
Taşkın Padır \\ College of Engineering \\ Northeastern University \\ t.padir@northeastern.edu
}

\begin{abstract}
Assistive service robots have a great potential for helping elderly or motor-impaired people in everyday tasks. Specifically, enabling robots to manipulate objects in home environments is a critical step towards independent life. In this work, we focus on developing a complete system for autonomous mobile manipulation. We describe our system, which consists of natural language processing, perception, navigation, and integrated motion and grasp planning modules.
\end{abstract}

\section{CCS CONCEPTS}

- Computer systems organization $\rightarrow$ Robotic autonomy.

\section{KEYWORDS}

Service robotics, Mobile manipulation

\section{ACM Reference Format:}

Tarık Keleştemur, Naoki Yokoyama, Joanne Truong, Anas Abou Allaban, and Taşkın Padır. 2019. System Architecture for Autonomous Mobile Manipulation of Everyday Objects in Domestic Environments. In The 12th PErvasive Technologies Related to Assistive Environments Conference (PETRA '19), June 5-7, 2019, Rhodes, Greece. ACM, New York, NY, USA, 6 pages. https://doi.org/10.1145/3316782.3316797

\section{INTRODUCTION}

According to the U.S. Census Bureau's report [9], 9 percent of the world population is 65 years old or older. This number is expected to increase to 12 percent by 2030 , and to 17 percent by 2050 . This rapid growth of older population will lead to a more severe lack of eldercare due to limited government resources and expensive eldercare services. One of the most important features of robotic eldercare would be autonomous manipulation of everyday objects. In [1], the authors conducted a survey from user perspectives to

Permission to make digital or hard copies of all or part of this work for personal or classroom use is granted without fee provided that copies are not made or distributed for profit or commercial advantage and that copies bear this notice and the full citation on the first page. Copyrights for components of this work owned by others than ACM must be honored. Abstracting with credit is permitted. To copy otherwise, or republish, to post on servers or to redistribute to lists, requires prior specific permission and/or a fee. Request permissions from permissions@acm.org.

PETRA '19, fune 5-7, 2019, Rhodes, Greece

(C) 2019 Association for Computing Machinery.

ACM ISBN 978-1-4503-6232-0/19/06 ..\$15.00

https://doi.org/10.1145/3316782.3316797 build a comprehensive list of household tasks expected from service robots. The results show that the ability to manipulate objects is an essential skill for robots that operate in domestic environments. Moreover, in our previous work [17], we surveyed more than 300 people to understand the requirements of a personal assistance robot and concluded that object retrieval is among the highest desired skills. With recent developments in perception, motion planning, and speech recognition, it is possible now more than ever for service robots to help elderly people or people with motor impairments to be self-sufficient.

The majority of research in mobile manipulation has been focused on solving individual problems. Although there has been great success in motion planning, perception, grasping, and user interfaces for mobile manipulation, there are few holistic works that report on integrated, complete systems. In [11], the authors developed a tele-operated mobile manipulator for an object retrieval task. The user uses a joystick to command the robot and grasp objects. The design of the robot limits its ability to grasp only from the floor, and joystick-based tele-operation makes it impossible to grasp an object outside of the user's field of view. In another work [10], the authors developed a system capable of grasping objects autonomously from flat surfaces. The object of interest is selected using a laser pointer, limiting the workspace of the robot to the user's current environment. Furthermore, occlusions might make it hard for the user to successfully command the robot. The closest work to ours is [2] in which a commercial mobile manipulator, PR2, is enhanced to carry out autonomous mobile manipulation in homes. [2] shows a successful integration of motion planning, grasping, and navigation. However, it requires the user to move the gripper using a 3D interactive marker through a graphical user interface in order to manipulate objects, which makes the system harder to use for elderly people. In contrast, our system depends only on speech commands. Another successful work on complete mobile manipulation is reported in [23]. The authors present each component of the system in detail and how they integrated all of them together. Their system uses checkerboards to create a 3D map of the environment to use for manipulation. Although it gives an accurate model, it is a time-consuming method. Our system does not rely on previously generated 3D maps for manipulation. It is mentioned that their system has a speech recognition module, however, it is not clear how this is incorporated into the manipulation. 


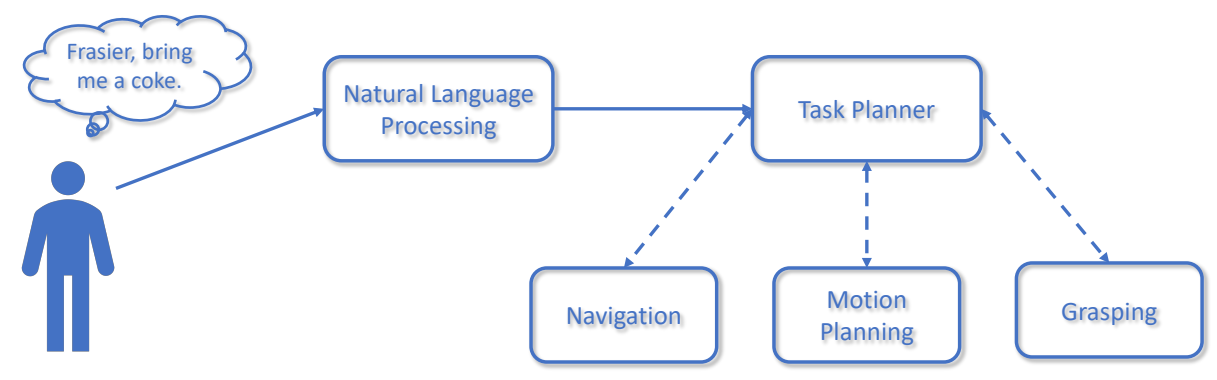

Figure 1: A diagram of the high-level system architecture.

For successful object manipulation in dynamic environments, the system should have the following features:

- a user-friendly interface,

- high-accuracy perception algorithms,

- robust motion planning and grasping algorithms, and

- the ability to navigate around safely.

In this paper, we present a system architecture to realize mobile manipulation in domestic environments. We describe our utilization of neural networks for object detection and segmentation, our grasp synthesis and motion planning approach, the mapping and planning for navigation, our speech recognition and understanding techniques, and finally the integration of all of these modules.

\section{METHODOLOGY}

\subsection{System Overview}

Our development platform used in this study is Toyota's Human Support Robot (HSR). The HSR is a mobile service robot equipped with a 5 degrees-of-freedom (DOF) arm, a parallel-jaw gripper, and a 3 DOF omni-drive base. The HSR's head has 2 DOF, one pair of stereo cameras, a wide angle RGB camera, and a depth sensor. The base of the HSR is modeled as two prismatic and one revolute joint, and is combined with the arm to model an 8 DOF whole-body chain.

The flowchart of the complete system can be seen in Figure 1. The HSR continuously listens for a user command and starts processing the audio when it detects the hot word, 'Frasier'. The natural language processing (NLP) module analyzes the remaining audio stream and outputs three attributes regarding the manipulation task: the name of the object, the location of the object, and the task itself. The task could be searching for an object (if the location is not known), bringing the object back to the user or a known location, or counting the amount of objects. The HSR has prior knowledge of the objects' approximate locations. Once the user command is analyzed, the task planner module uses these attributes to create a set of robot actions. If the object of interest is not in the HSR's scene, it starts to navigate to the most probable location of the object. If the user provided a location for the object, the HSR uses this location. After it locates the object, it can execute the grasping task and return to the operator or place the object in a predefined location, e.g., a bin.

All of the modules in the system are integrated into the Robot Operating System (ROS) [18] for fast development and easy debugging. For environment modeling and collision checking, the OpenRAVE simulation platform [3] is used.

\subsection{Natural Language Processing}

To translate speech into discretized tasks for the robot, we utilize a combination of various neural networks (NN) specialized for natural language processing (NLP). First, we use Google's Speech NN API [7] in order to convert audio into text, and to detect hot words to notify the robot that a command is being given. We then use Google's NLP NN API [7] to assign each word in the sentence a label (i.e., verb, location, pronoun, etc.) based on the context provided by the sentence (noun vs. verb, ex. the milk vs. to milk). We use this to construct a sequence of tasks for the robot to perform, using verbs as a cue to determine the robot task type and the nouns as cues for locations or corresponding object classes. To classify verbs and nouns that are not explicitly in our dictionary, we use Google's Word2Vec NN [15] to match it to a similar verb in our dictionary. To do this, we compute the cosine between the input word's vector representation to each of the vectors of the words in our dictionary, and check whether or not the largest cosine computed passes a certain threshold. To determine whether or not a perceived sentence is a special predefined command, we use the Levenshtein distance to compare it to our list of known pre-defined special commands. Tasks extract related information from the HSR's sensors and known state of the environment during execution to determine feasibility and success.

\subsection{Navigation}

We use the Dynamic Window Approach (DWA) [5] algorithm for local trajectory planning and Dijkstra's algorithm [4] with an $A^{*}$ heuristic for global trajectory planning. These algorithms are built into the ROS navigation stack, making them reliable path planners for navigating in a home environment. Hector SLAM [12] is utilized to generate a $2 \mathrm{D}$ map of the environment. The NLP module is used to store environment locations (e.g., kitchen) at the request of the operator in this generated map.

\subsection{Perception}

Our perception framework consists of two main modules: (I) object detection and pixel-wise segmentation to localize objects of interest and (II) point cloud processing that is used for modeling the environment and objects for motion planning and grasping.

2.4.1 Object Detection and Segmentation. We use the Mask R-CNN framework [8] for object detection and segmentation. In order to gather a sufficiently large amount of training data in an efficient 
and rapid manner, our approach involves generating an exhaustive artificial training set afflicted with various types of noise. By adding noise that may appear in the images taken by the camera, we train our object detection model to be more robust against them during inference [24].

To generate the artificial training images, videos of each object rotating atop an automatic turntable are recorded. This is done at various camera elevations to capture all angles of the object from different perspectives. Background subtraction is then performed on each frame of the video in order to extract all contours of the object. These extracted contours are then randomly chosen, scaled, and rotated, before being placed on an image of scenery similar to the types of environments the HSR may operate in. For each image, randomly chosen contours from a few randomly chosen classes are used in order to teach our model how to best distinguish the objects from each other when they appear together, which is especially helpful for objects that are similar in appearance. Occlusions, up to a certain percentage threshold, are allowed and are properly recorded when annotations are created. Each of these composite images are then afflicted with random adjustments in lighting and artificial image noise. Once a composite is generated, a corresponding annotation that records the object instance's class and the pixel-wise contour is created in COCO format [13].

Our object detection application was also extended by implementing OSLSM [22], a low-shot learning method for semantic segmentation that allowed us to train only on a few images without having to collect an exhaustive dataset. This helped us to instantly teach the robot to recognize objects it had never seen before.

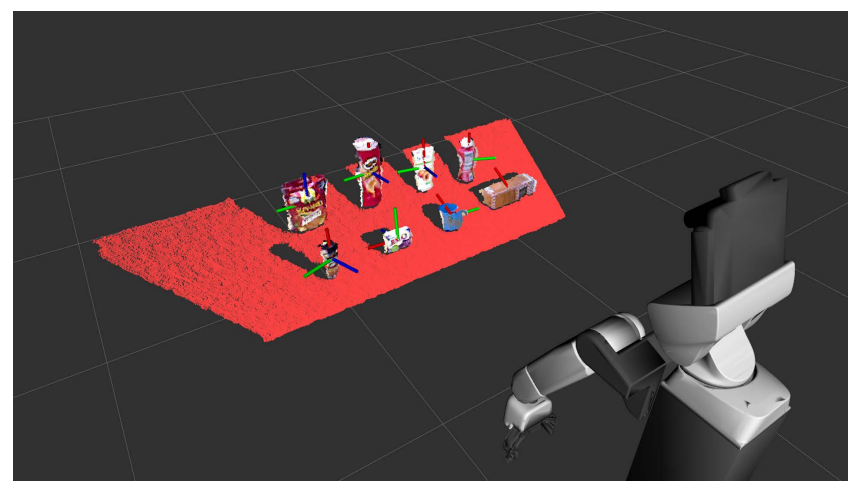

Figure 2: Tabletop Object Clustering. Red: segmented table plane RGB: object clusters.

2.4.2 Point Cloud Processing. In order to plan collision-free trajectories, a complete model of the environment needs to be generated. The processing starts with down-sampling the raw point cloud. Later, we use the RANSAC algorithm implemented in [19] to segment planes in the point cloud (e.g., shelf racks, table). After segmenting primitive shapes, we use Hierarchical Approximate Convex Decomposition method [14] to generate meshes of the remaining point cloud in the scene. For estimating the shape and size of the objects, we implement various algorithms. Once the object of interest is segmented in the RGB domain using Mask R-CNN, the correspondence between the RGB and the depth sensor is used to get point cloud cluster of the object. The object cluster is then fed into the RANSAC algorithm to estimate its primitive shape. The position and orientation of the object cluster is found using principal component analysis as explained in [20] which is then used for grasp synthesis. Figure 2 shows the result of the complete point cloud processing and the HSR model.

\subsection{Grasping and Motion Planning}

2.5.1 Grasping. A heuristic-based grasp synthesis method that uses the geometries of the object and the HSR's gripper is developed. The method takes the object's primitive shape, size and the transformation as the input. A number of grasp poses that are aligned with the principal axes of the object are sampled uniformly over the surface of the object. Pre-grasp positions are found by shifting the sampled points outside of the object along the principal axes by $5 \mathrm{~cm}$. These pre-grasp poses are then filtered out by checking collisions with the environment. Finally, the closest grasp pose to the HSR's gripper is selected.

2.5.2 Motion Planning. The motion planning problem can be formulated as a non-convex optimization problem subject to inequality and equality constraints and can be solved by a nonlinear optimization algorithm such as sequential quadratic programming (SQP). In this study, the decision variables are the joint positions for $T$ time steps, and the cost function is the distance between consecutive configurations:

$$
f\left(q_{1: T}\right)=\sum_{1}^{T}\left\|q_{t+1}-q_{t}\right\|^{2},
$$

where $q_{t} \in R^{8}$ represents the joint configuration at the $t$-th time step for the 8 DOF kinematic chain. The desired end-effector pose is enforced by an equality constraint such that:

$$
\triangle F K\left(q_{t}\right)=0,
$$

where $F K$ is the forward kinematics function that calculates the Cartesian end-effector pose given joint positions and $\Delta F K\left(q_{T}\right)=$ $\left[\Delta x_{t}, \Delta y_{t}, \Delta z_{t}, \Delta\right.$ roll $_{t}, \Delta$ pitch $\left._{t}, \Delta y a w_{t}\right]$ is the deviation of the endeffector position and Euler angles from the desired pose. The joint limits are defined as inequality constraints:

$$
\left(q_{t}-q^{-}\right)>0 \text { and }\left(q^{+}-q_{t}\right)>0
$$

where $q^{+}$and $q^{-}$are the maximum and minimum limits of the joint positions, respectively. The Gilbert-Johnson-Keerthi algorithm [6] is used to compute the collision distance, and a hinge-loss function is used to set up the constraints as described in [21]. Once a trajectory is planned, it is smoothed using tension spline interpolation based on the velocity and acceleration limits. Finally, the resulting trajectory is sent to the HSR's position controller as a reference.

\section{RESULTS}

Here, we discuss the performance of the perception and motion planning modules. We also report the results of our system performance in a realistic home environment. We chose the annual RoboCup@Home competition [25] as a test-bed. Benchmarking robotic research, especially manipulation systems, is a challenging problem because of high variations in environmental conditions 
Table 1: Storing Groceries Task Results

\begin{tabular}{ccl}
\hline Year & Number of Objects & Completion Time [min] \\
\hline 2017 & 1 & 5 \\
2018 & 6 & 3.41 \\
\hline
\end{tabular}

and the lack of standardized experiment setups. The robotic competitions are becoming increasingly popular to solve this by providing an uncontrolled environment and letting the participants demonstrate their approaches in the same conditions. On the other hand, the RoboCup@Home competition provides a very realistic home environment for various tasks including speech recognition, object manipulation, navigation, and human-robot interaction (HRI). There are 3 sub-leagues under the @Home leagues: domestic standard platform (DSPL), Social Standard Platform (SSPL) and Open Platform leagues (OPL). We participated in the DPSL, in which participants use the Toyota HSR as the standard platform.

\subsection{System Performance}

One of the most challenging tasks in the RoboCup@Home competition is the Storing Groceries task ${ }^{1}$ in which the robot has to grasp objects from a table and put them onto a shelf. Similar objects (objects that are in the same category) needs to be put together on the shelf. The tasks start with a speech command. The robot needs to finish the task within 5 minutes and pick and place 5 objects in total.

The storing groceries task is a great experiment case for our system since it requires all modules we have developed to work in an integrated manner. It is important to note that the configurations of environment is not known beforehand and the participants have only 2 hour access to the objects. The Table 1 shows our results of the storing groceries task in the last two years. In RoboCup@Home 2018, we were the only team in the DSPL to complete the task in time and won the Best Manipulation Skills award. ${ }^{2}$
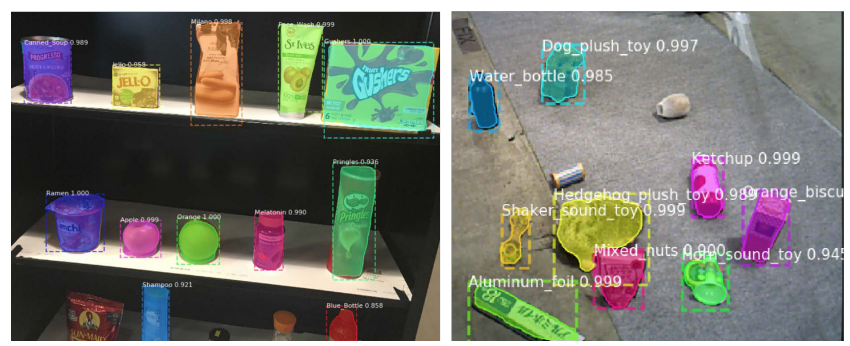

Figure 3: Results of our artificially trained model on real images. Right image shows the robustness to orientation and occlusion.

\subsection{Perception Performance}

Several tens of thousands of composites are created and split to form both a training set and a validation set for our object segmentation neural network. As a result, after training, our model

\footnotetext{
${ }^{1}$ The details of the task can be found at https://github.com/RoboCupAtHome/RuleBook ${ }^{2}$ The video of our run can be found at https://youtu.be/a1MAy_SDU7k
}
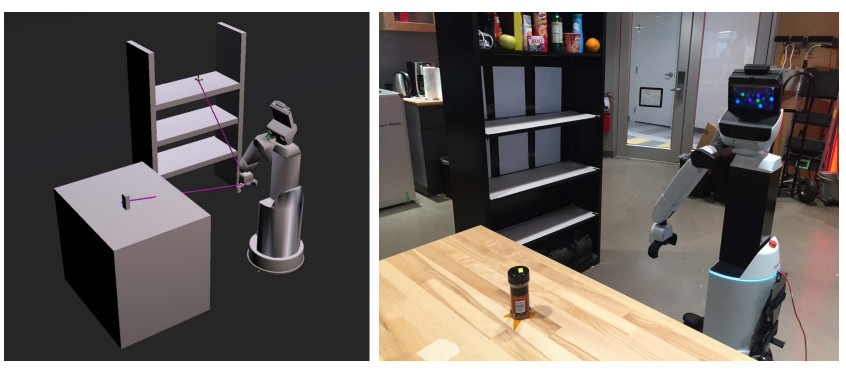

Figure 4: Experiment setup: (Left) Environment model (Right) Real world

demonstrates 92.6 percent accuracy in detecting objects regardless of size, proximity to the camera, orientation, occlusions, lighting, and noise as can be seen in Figure 3.

\subsection{Motion Planning Performance}

To evaluate the performance of our motion planning method, we conducted a real world pick-and-place task experiment. The overall experiment is picking an object from a table and placing it onto a shelf within the robot's workspace. The experimental setup is shown in Figure 4. The pick position is selected $3 \mathrm{~cm}$ away from the object's center towards the robot and the orientation is aligned with the object's principal axes. The place pose is selected as the middle of the top shelf rack. For a fair comparison, the environment is modeled in the beginning and used for all tests. Two cases are tested:

3.3.1 Two-step Planning. A classical two-step approach in which two separate trajectories are planned for each of the picking and placing poses. The robot executes the first plan, grasps the object, executes the second plan, and places the object. For each trajectory plan, 5 waypoints are used with the end-effector goals given used as a pose constraint at the last waypoint.

3.3.2 One-step Planning. The whole task is planned in one step where 10 waypoints are used and the pick and place pose are given as pose constraints at the 5 th and 10 th waypoints, respectively. Since the robot will be moving at the picking waypoint, an external controller is used to check whether the end-effector has reached the picking pose by looking at the difference of current end-effect pose and goal pose. If the error is less than $10^{-4}$, the fingers are closed immediately. Each case is executed 15 times.

The two cases are compared in terms of planning time, total path length and the execution time. No notable improvements have been observed for the planning time (around 1 second for both cases) and the path length, however, a significant improvement has been recorded for the execution time. For one-step planning, the mean of the execution time over 15 pick-and-place tasks is found to be 20.15 seconds whereas for the two-step planning, it is 26.96 seconds. The standard deviation and mean of execution times are depicted in Figure 5. Since we are using the sum of joint displacements for each consecutive waypoint as the cost function (1), the subsequent joint configurations after picking are close to the picking configuration when one-step planning is used. Thus, it is faster for the robot to go from the picking configuration to the placing configuration. Also, 
one-step planning produces a complete trajectory which results in a better velocity profiling during trajectory smoothing.

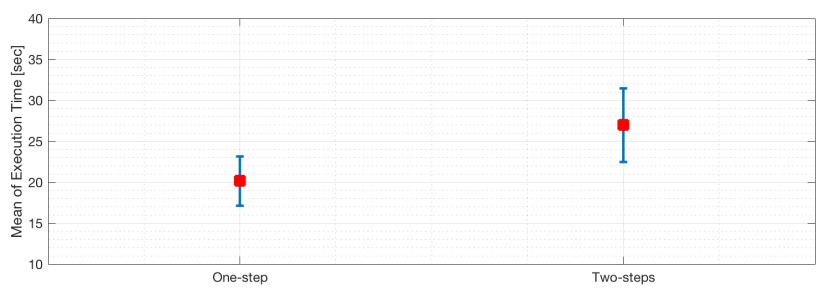

Figure 5: Mean and standard deviation of execution times

\section{CONCLUSIONS}

Assistive robots will be a major factor for helping humans and improving lives in the near future. To this end, we have developed and deployed a successful mobile manipulation system and showed its capabilities in a realistic home environment. The system integration and details of each module are explained in details. Note that that the structure of our system is modular and each module can easily be applied to any robot.

Although we have successful experiments in our lab and even in realistic home environments, there are a number of advancements that need to be done to deploy these robots into real homes. First, the NLP frameworks do not work very well with different accents and dialects. A multi-language and multi-dialect NLP framework should be considered for these platforms. Second, data collection and training for deep learning models for object detection and segmentation require an expert and often take several hours. There are methods [22] that learn using a small number of images, but the robot should have an intuitive way of getting images of the objects without an expert operator's help. Third, navigation in tight and confined spaces can be challenging even with the state-of-the-art algorithms. To be able to move objects around and make space for the navigation can potentially solve this problem. Finally, during our lab tests and RoboCup@Home runs, we have observed that our grasping methods fail in highly cluttered environments due to three encountered problems: (I) The object detection and segmentation give low accuracy because of large occlusions. (II) The motion planning method cannot find a feasible trajectory. (III) Overlapped objects make it impossible the grasp the object of interest. For the first issue, the perception algorithm should reason about low accuracy and move the robot towards better viewpoints. To overcome the second and third issues, the robot needs to interact with the world e.g. pushing the objects away to reach the object of interest. Thus, a non-prehensile manipulation method such as [16] needs to be integrated into the system.

Our future work includes developing an integrated non-prehensile and grasping method as well as a re-planning approach to account for the disturbances during the trajectory execution. We are also working on an affordance-based navigation method to be able to move objects around and navigate in cluttered spaces.

\section{ACKNOWLEDGMENTS}

This material is based upon work supported by the National Science Foundation under Award No. 1649729 and Toyota Motor Corporation.

\section{REFERENCES}

[1] Maya Cakmak and Leila Takayama. 2013. Towards a comprehensive chore list for domestic robots. In Proceedings of the 8th ACM/IEEE international conference on Human-robot interaction. IEEE Press, 93-94.

[2] Matei Ciocarlie, Kaijen Hsiao, Adam Leeper, and David Gossow. 2012. Mobile manipulation through an assistive home robot. In Intelligent Robots and Systems (IROS), 2012 IEEE/RSf International Conference on. IEEE, 5313-5320.

[3] Rosen Diankov and James Kuffner. 2008. Openrave: A planning architecture for autonomous robotics. Robotics Institute, Pittsburgh, PA, Tech. Rep. CMU-RI-TR-083479 (2008).

[4] Edsger W Dijkstra. 1959. A note on two problems in connexion with graphs. Numerische mathematik 1, 1 (1959), 269-271.

[5] Dieter Fox, Wolfram Burgard, and Sebastian Thrun. 1997. The dynamic window approach to collision avoidance. IEEE Robotics \& Automation Magazine 4, 1 (1997), 23-33.

[6] Elmer G Gilbert, Daniel W Johnson, and S Sathiya Keerthi. 1988. A fast procedure for computing the distance between complex objects in three-dimensional space. IEEE Journal on Robotics and Automation 4, 2 (1988), 193-203.

[7] Google. 2019. Cloud Natural Language API. Retrieved January 22, 2019 from https://cloud.google.com/natural-language/

[8] Kaiming He, Georgia Gkioxari, Piotr Dollár, and Ross Girshick. 2017. Mask $\mathrm{r}$-cnn. In Computer Vision (ICCV), 2017 IEEE International Conference on. IEEE, 2980-2988.

[9] Wan He, Daniel Goodkind, and Paul R Kowal. 2016. An aging world: 2015. United States Census Bureau Washington, DC.

[10] Advait Jain and Charles C Kemp. 2010. EL-E: an assistive mobile manipulator that autonomously fetches objects from flat surfaces. Autonomous Robots 28, 1 (2010), 45.

[11] Chih-Hung King, Tiffany L Chen, Zhengqin Fan, Jonathan D Glass, and Charles C Kemp. 2012. Dusty: an assistive mobile manipulator that retrieves dropped objects for people with motor impairments. Disability and Rehabilitation: Assistive Technology 7, 2 (2012), 168-179.

[12] S. Kohlbrecher, J. Meyer, O. von Stryk, and U. Klingauf. 2011. A Flexible and Scalable SLAM System with Full 3D Motion Estimation. In Proc. IEEE International Symposium on Safety, Security and Rescue Robotics (SSRR). IEEE.

[13] Tsung-Yi Lin, Michael Maire, Serge J. Belongie, Lubomir D. Bourdev, Ross B. Girshick, James Hays, Pietro Perona, Deva Ramanan, Piotr Dollár, and C. Lawrence Zitnick. 2014. Microsoft COCO: Common Objects in Context. CoRR abs/1405.0312 (2014). arXiv:1405.0312 http://arxiv.org/abs/1405.0312

[14] Khaled Mamou and Faouzi Ghorbel. 2009. A simple and efficient approach for 3D mesh approximate convex decomposition. In Image Processing (ICIP), 2009 16th IEEE International Conference on. IEEE, 3501-3504.

[15] Tomas Mikolov, Ilya Sutskever, Kai Chen, Greg S Corrado, and Jeff Dean. 2013. Distributed representations of words and phrases and their compositionality. In Advances in neural information processing systems. 3111-3119.

[16] A. O. Onol, P. Long, and T. Padır. 2018. A Comparative Analysis of Contact Models in Trajectory Optimization for Manipulation. In 2018 IEEE/RSf International Conference on Intelligent Robots and Systems (IROS). 1-9. https://doi.org/10.1109/ IROS.2018.8594284

[17] T. Padir, J. Skorinko, and V. Dimitrov. 2015. User-centric design of a personal assistance robot (FRASIER) for active aging. In 2015 37th Annual International Conference of the IEEE Engineering in Medicine and Biology Society (EMBC). 50205023. https://doi.org/10.1109/EMBC.2015.7319519

[18] Morgan Quigley, Ken Conley, Brian Gerkey, Josh Faust, Tully Foote, Jeremy Leibs, Rob Wheeler, and Andrew Y Ng. 2009. ROS: an open-source Robot Operating System. In ICRA workshop on open source software, Vol. 3. Kobe, Japan, 5.

[19] Radu Bogdan Rusu and Steve Cousins. 2011. 3d is here: Point cloud library (pcl). In Robotics and Automation (ICRA), 2011 IEEE International Conference on. IEEE, $1-4$.

[20] Radu Bogdan Rusu, Zoltan Csaba Marton, Nico Blodow, Mihai Dolha, and Michael Beetz. 2008. Towards 3D point cloud based object maps for household environments. Robotics and Autonomous Systems 56, 11 (2008), 927-941.

[21] John Schulman, Yan Duan, Jonathan Ho, Alex Lee, Ibrahim Awwal, Henry Bradlow, Jia Pan, Sachin Patil, Ken Goldberg, and Pieter Abbeel. 2014. Motion planning with sequential convex optimization and convex collision checking. The International fournal of Robotics Research 33, 9 (2014), 1251-1270.

[22] Amirreza Shaban, Shray Bansal, Zhen Liu, Irfan Essa, and Byron Boots. 2017. One-Shot Learning for Semantic Segmentation. CoRR abs/1709.03410 (2017). arXiv:1709.03410 http://arxiv.org/abs/1709.03410 
[23] Siddhartha S Srinivasa, Dave Ferguson, Casey J Helfrich, Dmitry Berenson, Alvaro Collet, Rosen Diankov, Garratt Gallagher, Geoffrey Hollinger, James Kuffner, and Michael Vande Weghe. 2010. HERB: a home exploring robotic butler. Autonomous Robots 28, 1 (2010), 5 .

[24] Joshua Tobin, Rachel Fong, Alex Ray, Jonas Schneider, Wojciech Zaremba, and Pieter Abbeel. 2017. Domain Randomization for Transferring Deep Neural Networks from Simulation to the Real World. CoRR abs/1703.06907 (2017) arXiv:1703.06907 http://arxiv.org/abs/1703.06907

[25] Thomas Wisspeintner, Tijn Van Der Zant, Luca Iocchi, and Stefan Schiffer. 2009 RoboCup@ Home: Scientific competition and benchmarking for domestic service robots. Interaction Studies 10, 3 (2009), 392-426. 